\title{
Terceiro molar incluso fusionado com supranumerário: Relato de caso
}

\author{
Included third molar fused with supernumerary: Case report \\ Tercer molar incluído fusionado con supernumerario: Reporte de caso
}

\section{Resumo}

A fusão é uma anomalia dentária caracterizada pela união de dois germes dentários. Os dentes supranumerários são elementos que se desenvolvem além da dentição normal. A combinação desses dois desvios dos padrões de normalidade torna o diagnóstico desafiador. Essas anomalias podem resultar em graves problemas no desenvolvimento da dentição se não forem diagnosticadas e tratadas precocemente. Após um correto diagnóstico, o cirurgião dentista deve avaliar todos os parâmetros e decidir qual a melhor conduta de tratamento. A remoção do dente é frequentemente indicada quando se apresenta com complicação ou qualquer patologia associada. O objetivo é relatar um caso de terceiro molar inferior fusionado com um supranumerário, discorrer sobre essas anomalias, bem como prevalência, manejo e discussão do tema. Trata-se de um relato de caso, do tipo qualitativo descritivo, com embasamento na literatura científica. A literatura mostra que a fusão entre um supranumerário e um outro elemento é uma condição rara, e em alguns casos pode-se optar pelo tratamento endodôntico, mas em outros é viável o cirúrgico. No caso relatado, optou-se pela remoção cirúrgica do elemento, a fim de prevenir uma impacção do dente quando completamente desenvolvido, o que dificultaria ainda mais o tratamento. Concluímos que o acompanhamento da evolução dos terceiros molares deve ser feito desde cedo para observar seu processo de formação e diagnosticar qualquer anomalia que venha a surgir, a fim de solucionar precocemente e evitar maiores consequências. Ademais, ressaltamos a importância da TCFC no diagnóstico diferencial e na obtenção de informações para o planejamento cirúrgico.

Palavras-chave: Terceiro molar; Fusão; Supranumerário; Anomalias dentárias.

\begin{abstract}
Fusion is a dental anomaly characterized by the union of two tooth germs. Supernumerary teeth are elements that develop beyond normal dentition. The combination of these two deviations from normal standards makes diagnosis challenging. These anomalies can result in serious problems in dentition development if they are not diagnosed and treated early. After a correct diagnosis, the dental surgeon must evaluate all parameters and decide on the best treatment approach. Tooth removal is often indicated when it presents with complications or any associated pathology. The objective is to report a case of a mandibular third molar fused with a supernumerary, discuss these anomalies, as well the prevalence, management, and discussion of the subject. This is a case report, of the descriptive qualitative type, based on the scientific literature. The literature shows that the fusion between a supernumerary and another element is a rare condition, and in some cases, endodontic treatment can be chosen, but in others surgical treatment is feasible. In the case reported here, we opted for surgical removal of the element to prevent impaction of the tooth when fully developed, which would make the treatment even more difficult. We conclude that monitoring the evolution of third molars should be done early to observe their formation process and diagnose any anomaly that may arise, to resolve it early and avoid further consequences. Furthermore, we emphasize the importance of CBCT in differential diagnosis and in obtaining information for surgical planning.
\end{abstract}

Keywords: Third molar; Fusion; Supernumerary teeth; Dental anormalities. 


\begin{abstract}
Resumen
La fusión es una anomalía dental caracterizada por la unión de dos gérmenes dentales. Los dientes supernumerarios son elementos que se desarrollan más allá de la dentición normal. La combinación de estas dos desviaciones de los estándares normales hace que el diagnóstico sea un desafío. Estas anomalías pueden resultar en serios problemas en el desarrollo de la dentición si no se diagnostican y tratan a tiempo. Después de un diagnóstico correcto, el cirujano dental debe evaluar todos los parámetros y decidir el mejor enfoque de tratamiento. La extracción dentaria suele estar indicada cuando presenta complicaciones o alguna patología asociada. El objetivo es reportar un caso de un tercer molar mandibular fusionado con un supernumerario, discutir estas anomalías, así como la prevalencia, manejo y discusión del tema. Se trata de un caso clínico, de tipo descriptivo cualitativo, basado en la literatura científica. La literatura muestra que la fusión entre un supernumerario y otro elemento es una condición rara, y en algunos casos se puede optar por el tratamiento endodóntico, pero en otros el tratamiento quirúrgico es factible. En el caso aquí reportado, optamos por la remoción quirúrgica del elemento con el fin de evitar la impactación del diente cuando esté completamente desarrollado, lo que dificultaría aún más el tratamiento. Concluimos que el seguimiento de la evolución de los terceros molares debe realizarse de forma precoz para observar su proceso de formación y diagnosticar cualquier anomalía que pueda surgir, con el fin de resolverla precozmente y evitar mayores consecuencias. Además, destacamos la importancia de la CBCT en el diagnóstico diferencial y en la obtención de información para la planificación quirúrgica.
\end{abstract}

Palabras clave: Tercer molar; Fusión; Supernumerario; Anomalías dentales.

\title{
1. Introdução
}

As anomalias dentárias de desenvolvimento podem ser causadas por complexas interações entre fatores genéticos e ambientais durante o processo de desenvolvimento dentário, em particular ao longo dos estágios de morfodiferenciação ou histodiferenciação durante a odontogênese. Este processo é multifatorial, multinível e multidimensional. (Laganà et al., 2017; Temilola et al., 2014).

Nesse sentido, a fusão é uma anormalidade de desenvolvimento infrequente na forma do dente, causada pela união de dois germes dentários adjacentes (Silva et al., 2018; Vinothkumar et al., 2017). Essa alteração pode afetar as dentições decídua e permanente, bem como os dentes supranumerários (Ley et al., 2019). Dependendo do estágio de desenvolvimento do dente, a fusão pode ser classificada como completa ou incompleta; o primeiro envolve a raiz e a coroa, enquanto o último envolve apenas a coroa. Sua etiologia é incerta, mas as evidências mostram que fatores hereditários desempenham um papel importante (Ley et al., 2019).

Os dentes supranumerários são dentes que se desenvolvem além da dentição normal, apresentando-se já erupcionados ou não (Báez-quintero et al., 2017). Sua etiologia não é bem compreendida, mas várias hipóteses foram formuladas para explicar sua ocorrência, como à dicotomia do botão dentário, hiperatividade da lâmina dentária, ou a combinação de fatores genéticos e ambientais (Park et al., 2020; Silva et al., 2018). A frequência de desenvolvimento de dentes supranumerários na dentição permanente é de $0,15 \%$ a 3,9\% em caucasianos e superior a 3\% em indivíduos de raça mongolóide. De acordo com Shah et al. (2015) podem afetar mandíbula ou maxila, uni ou bilateralmente, com a presença de um único ou até mesmo vários supranumerários. O planejamento e o momento do tratamento dependem da localização, do estado de erupção e da presença de complicações (Park et al., 2020).

Assim, a combinação desses dois desvios dos padrões de normalidade torna o diagnóstico desafiador, pois não altera o número de dentes no arco (Silva et al., 2018). Essas anomalias podem resultar em graves problemas no desenvolvimento da dentição se não forem diagnosticadas e tratadas precocemente (Moreira et al., 2016; Saberi et al., 2016). Diante disso, a tomografia computadorizada de feixe cônico (TCFC) é um exame imprescindível para fornecer as informações necessárias para o diagnóstico e planejamento cirúrgico nos casos em que as radiografias convencionais não revelam informações conclusivas (Vinothkumar et al., 2017; Smail-Faugeron et al., 2016; Castro et al., 2014).

Dessa forma, após um correto diagnóstico, o cirurgião dentista deve avaliar todos os parâmetros e decidir qual será a melhor conduta de tratamento (Stringhini et al., 2015; Mohan et al., 2012). A remoção do dente é frequentemente indicada quando se apresenta com complicação ou qualquer patologia associada (Roslan et al., 2020; Dias et al., 2019). O objetivo deste 
trabalho é relatar um caso de terceiro molar inferior fusionado com um supranumerário, discorrer sobre essas anomalias, bem como prevalência, manejo e discussão do tema.

\section{Metodologia}

O presente estudo trata-se de um relato de caso clínico, do tipo qualitativo descritivo (Pereira et al., 2018), com embasamento na literatura científica. Foi realizada a pesquisa de artigos científicos nos seguintes portais e bases de dados: National Library of Medicine (PubMed)/MEDLINE, Google Scholar, Scientific Electronic Library Online (Scielo) e Biblioteca Virtual de Saúde (BVS).

Além disso, foram seguidos os princípios éticos de pesquisa envolvendo seres humanos, sendo preservada a identidade da voluntária e fornecido esclarecimentos sobre o tratamento. Os procedimentos clínicos foram iniciados somente após o paciente concordar e assinar o Termo de Consentimento Livre e Esclarecido (TCLE), autorizando o tratamento, a divulgação das imagens e de dados coletados durante o atendimento clínico e os que constavam no prontuário, sendo que a mesma poderia desistir da participação no estudo a qualquer momento.

\section{Relato de Caso}

Paciente M.L.R.F, do sexo feminino, 18 anos, sem doenças de base e ou alergias, buscou atendimento odontológico em uma clínica particular do interior do Rio Grande do Norte com intuito de acompanhar o desenvolvimento dos terceiros molares presentes em sua na cavidade bucal, bem como realizar exodontias mediante necessidade.

Foi solicitado o exame radiográfico panorâmico e observou-se uma alteração na imagem do Terceiro Molar Inferior esquerdo ( $3^{\circ} \mathrm{MIE}$ ), sendo sugestivo de uma variação anatômica do elemento ou de um odontoma composto (por causa da semelhança das características radiográficas). Devido ao fato de o elemento estar incluso, totalmente recoberto por tecido mole e osso, e com uma certa proximidade com o canal mandibular, foi solicitada uma Tomografia Computadorizada de Feixe Cônico (TCFC) para a melhor avaliação do elemento dentário e planejamento do caso.

Os resultados tomográficos mostraram tratar-se de uma fusão entre o $3^{\circ} \mathrm{MIE}$ e um dente supranumerário, ambos ainda em formação. $\mathrm{O}^{\circ} \mathrm{MIE}$ encontrava-se incluso e impactado, mesioangulado, ligeiramente inclinado para a lingual, com sua coroa apresentando íntima relação com o segundo molar inferior esquerdo, sua raiz ainda em formação (estágio 7 de Nolla), e apresentando também uma íntima relação com a cortical óssea vestibular. $\mathrm{O}$ exame também mostrou que a proximidade do elemento com o canal mandibular não traria riscos de lesão ao Nervo Alveolar Inferior (NAI) (devido ao dente ainda encontrarse em formação).

Dessa forma, na Figura 1 pode-se observar a avaliação clínica inicial, no qual o terceiro molar fusionado encontra-se completamente incluso em osso e tecido, não sendo possível a visualização da coroa clínica e/ou sinais de áreas abauladas. 
Figura 1. Imagem inicial do exame clínico intraoral. Observe a área de localização do supranumerário.

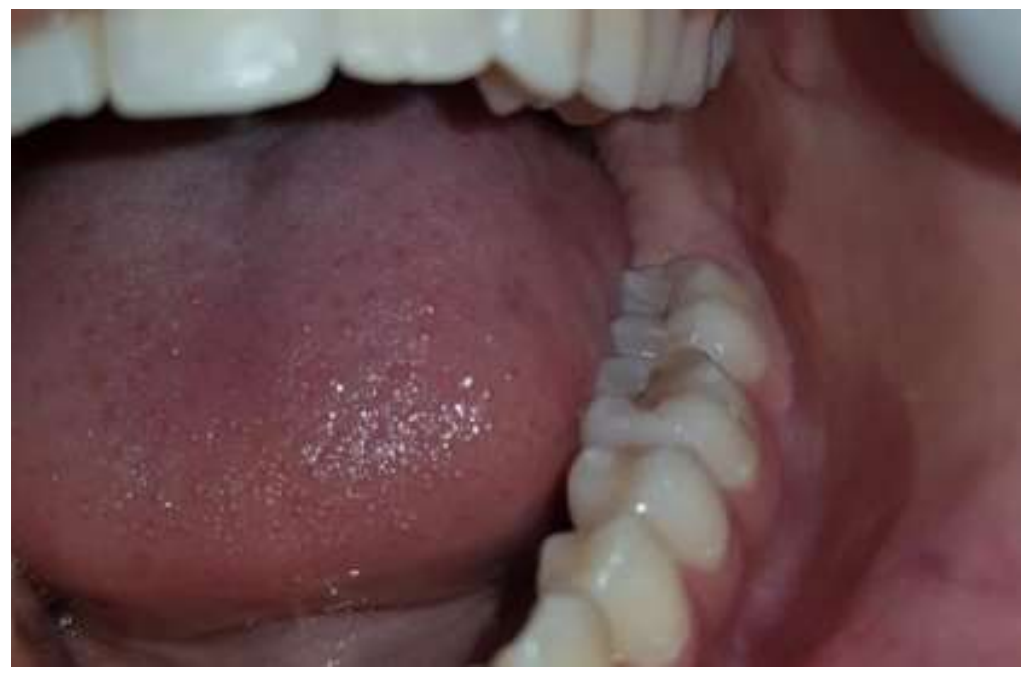

Fonte: Autores.

Na Figura 2, tem-se a imagem da radiografia panorâmica, a qual mostra uma característica anormal do terceiro molar inferior esquerdo, semelhante ao odontoma composto ( $1^{a}$ hipótese de diagnóstico), sobreposição com raízes do segundo molar inferior esquerdo e proximidade com canal mandibular. Diante disso, foi solicitada uma tomografia computadorizada cone-beam para melhor visualização.

Figura 2. Imagem radiográfica extraoral panorâmica. Observe a região distal do elemento dentário 37 (região mandibular direita da imagem), no qual o elemento supranumerário encontra-se fusionado com o terceiro molar.

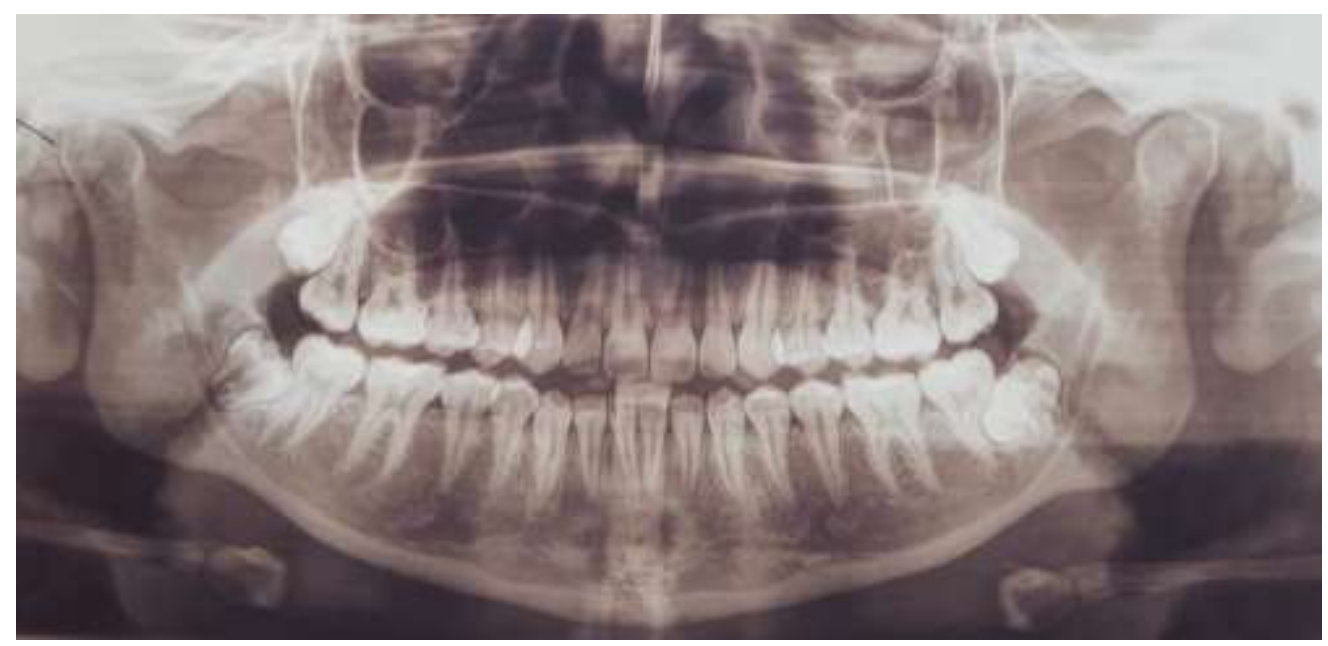

Fonte: Autores.

Na Figura 3, percebe-se a sequência das imagens obtidas com a tomografia computadorizada, recomendada para obter maiores detalhes do elemento. Assim, o exame confirma o diagnóstico de um terceiro molar inferior fusionado com um elemento supranumerário. Pode-se analisar que o terceiro molar anômalo se encontra em uma posição vestibular ao segundo molar inferior, em uma posição ectópica, e próximo ao Nervo Alveolar Inferior (NAI). 
Figura 3. Imagem 2D da tomografia computadorizada Cone Beam, utilizando cortes de $1 \mathrm{~mm}$ de espessura. Observe a proximidade dos elementos dentários ao canal mandibular.
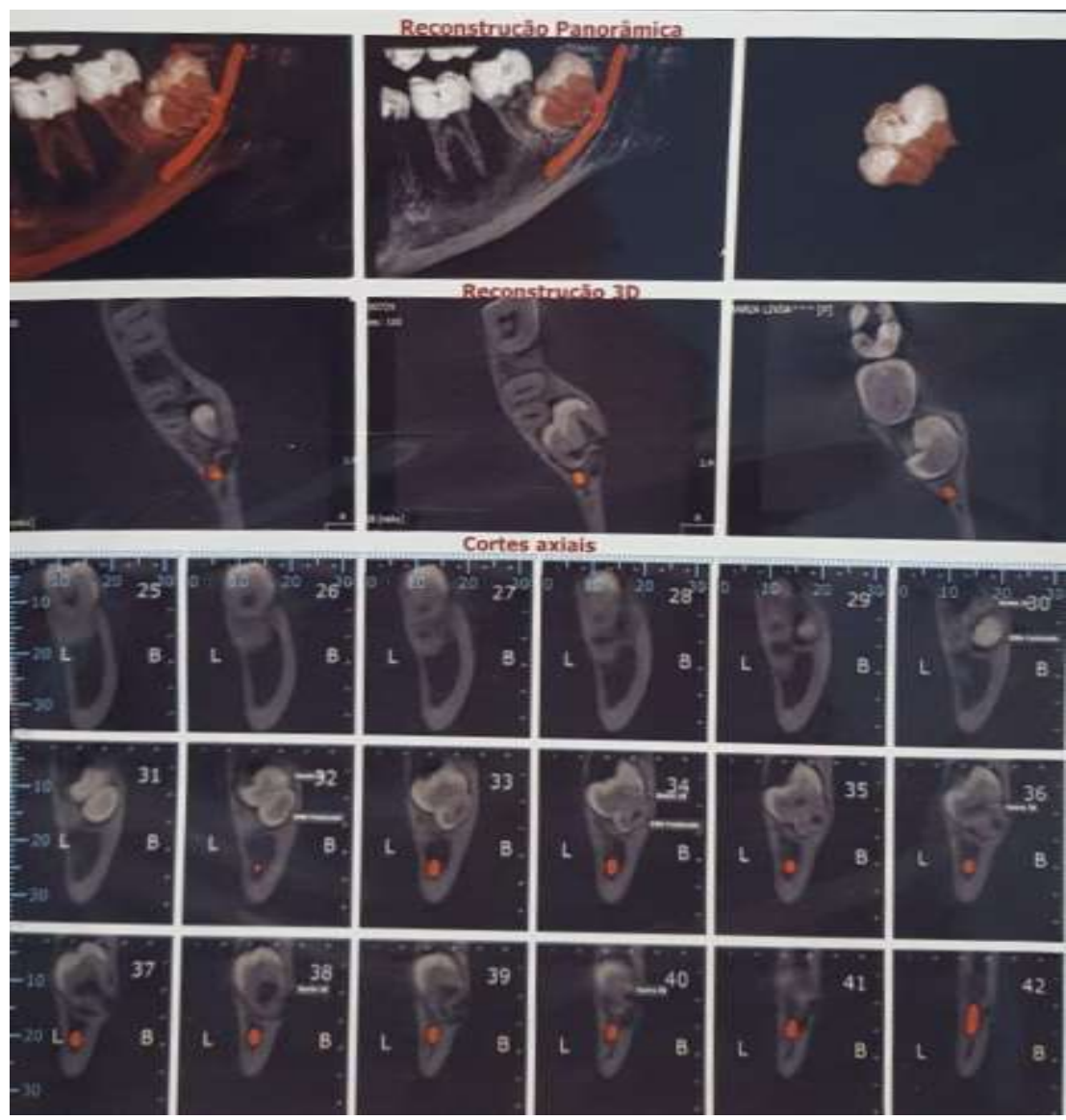

Fonte: Autores.

Após o planejamento do caso por meio das avaliações clínicas e radiográficas, optou-se pela remoção dos elementos dentários enquanto eles ainda estavam em formação. A escolha foi feita considerando que após o período de formação, poderiam surgir maiores complicações, como risco de fraturas e riscos de lesão ao NAI.

Após administrada a medicação para profilaxia antibiótica e analgesia preemptiva (amoxicilina, dexametasona e dipirona, administradas 1 hora antes do procedimento), deu-se início ao procedimento cirúrgico: a paciente foi submetida à anestesia local (mepivacaína 3\% com vasoconstritor felipressina), posteriormente, realizou-se a incisão de Ward expondo o osso mandibular que, ao ser desgastado, conseguiu-se o acesso e a melhor visualização do elemento que se encontrava completamente incluso. Foi realizada a osteotomia ao redor do elemento dentário com a finalidade de criar um alívio e permitir a adaptação da alavanca possibilitando a luxação e remoção do elemento.

Executada a extração dentária, seguiu-se para a regularização das margens ósseas da cavidade onde se alojava o dente, para evitar espículas ósseas, curetagem cuidadosa e irrigação da região. E em seguida, utilizou-se o Hemospon® para ajudar na manutenção e formação do coágulo. Foi realizada sutura com fio seda 4-0. A cirurgia seguiu seu curso normalmente, sem quaisquer intercorrências.

Na Figura 4, pode-se observar o momento transoperatório, no qual a letra A mostra uma visão após a incisão indicando posição do dente vestibularizado e incluso em osso; a letra B, uma visão do elemento após o desgaste ósseo; a letra C, a região onde se encontrava o elemento dentário; a letra D a cavidade com Hemospon ${ }^{\circledR}$ em seu interior; letra E, sutura; e a letra F, o elemento supranumerário fusionado com terceiro molar, em rizogênese incompleta, extraído de forma íntegra. 
A paciente seguiu corretamente a terapia medicamentosa prescrita para uso domiciliar (dexametasona 4mg, 1 comprimidos de 12/12 horas por 3 dias; dipirona 500mg 1 comprimido de $08 / 08$ horas por 03 dias) e os cuidados recomendados e, portanto, não houve complicação pós-operatória. Encontra-se hoje com 8 meses após a cirurgia, se recuperou bem, sem queixas e sem sequelas.

Figura 4. Fotografias intraorais do procedimento cirúrgico (observe todas as etapas do transoperatório). A. Incisão intraoral em região distal ao elemento dentário 37, estendendo-se até a papila mesial e afastamento do retalho. B. Descolamento do tecido, visualização da tábua óssea mandibular e visualização do terceiro molar fusionado ao supranumerário. C. Loja cirúrgica após a remoção dos elementos dentários. D. Preenchimento da cavidade com biomaterial. E. Sutura da área operada, com total união dos tecidos. F. Elementos dentários removidos.

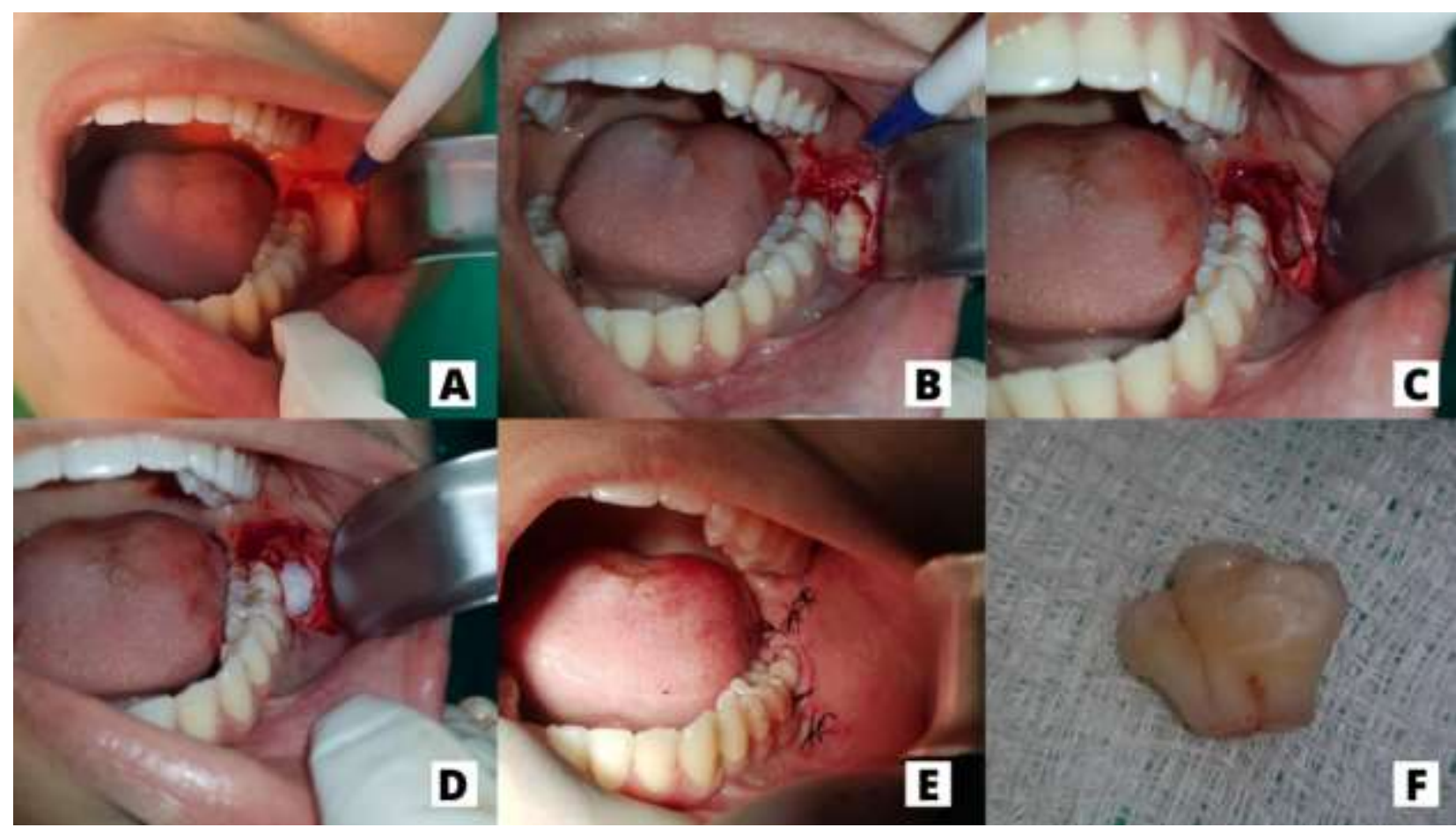

Fonte: Autores.

Na Figura 5, pode-se observar o momento do pós-operatório, com uma boa cicatrização e com o aspecto semelhante ao exame clínico inicial. 
Figura 5. Foto do pós-operatório de 8 meses. Observe a cicatrização tecidual local.

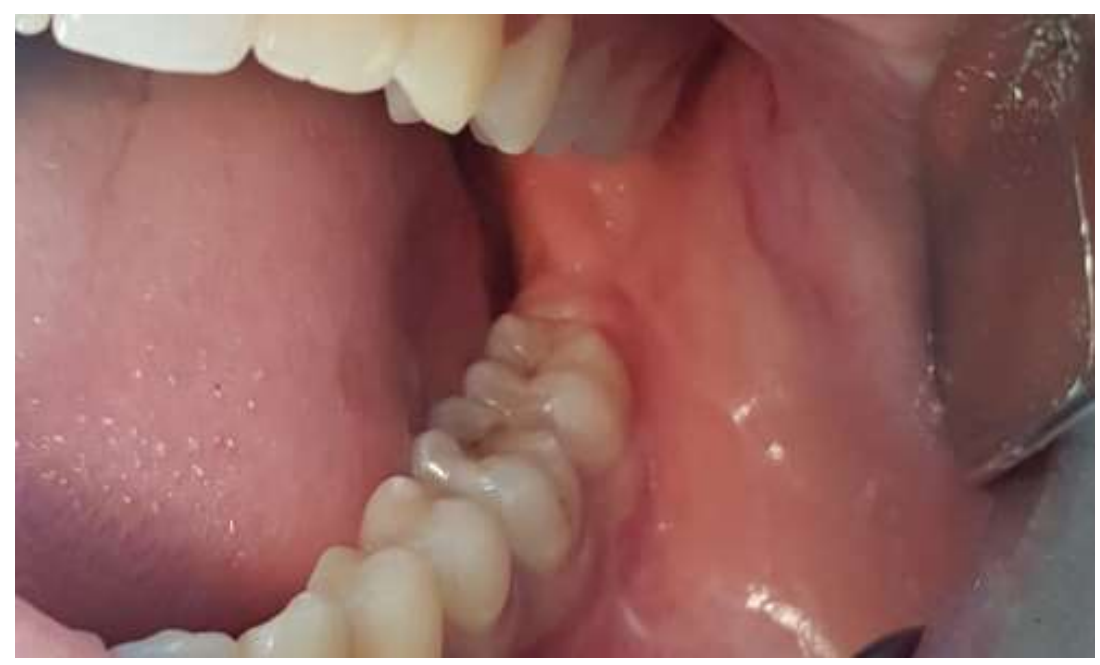

Fonte: Autores.

\section{Discussão}

De acordo com Park et al. (2020), em seu estudo de coorte retrospectivo, os dentes supranumerários foram menos prevalentes na região de mandíbula (apenas 1,0\% da amostra). A região posterior da mandíbula também é a região menos comum, apenas 47 dos 705 pacientes apresentaram dentes supranumerários nessa região, e aproximadamente $28 \%$ desses pacientes tiveram complicações durante a cirurgia. Magalhães et al. (2019) discorre em seu trabalho sobre a prevalência e frequência de alterações dentárias. Em sua pesquisa após a análise de 300 radiografias panorâmicas, encontrou uma prevalência de aproximadamente $13 \%$ de pacientes que apresentavam pelo menos um elemento supranumerário. No caso clínico exposto, a paciente apresentou um elemento supranumerário associado a outra anomalia dentária, a fusão.

Ley et al. (2020) mostraram em seu estudo, que a fusão dentária raramente afeta molares, e normalmente esses dentes fundidos causam problemas estéticos, ortodônticos e periodontais. Ferreira-Júnior et al. (2014) afirma que a fusão pode atingir de $0,5 \%$ a $1 \%$ da população, sendo apenas $0,05 \%$ a $0,2 \%$ encontrado na dentição permanente, e é mais comum na região de incisivos e caninos. Diante disso, é possível observar que as características encontradas na paciente em questão tornam o caso com o diagnóstico mais desafiador, por tratar-se de uma fusão entre um elemento da dentição permanente, na região de molar, e uma anomalia supranumerária.

Levando em consideração que as anomalias dentárias representam uma alteração tridimensional (3D) que pode ocorrer em toda a superfície dentária, uma investigação cuidadosa é necessária para obter um diagnóstico mais preciso e um tratamento adequado (Castro et al., 2014). No presente estudo, após a observação da radiografia panorâmica, o odontoma composto, um tumor odontogênico, tornou-se uma possível hipótese diagnóstica.

Segundo Neville et al. (2016), odontoma composto aparece como uma coleção de estruturas semelhantes a dentes de variados tamanhos e formas. Essas características são semelhantes ao que foi observado no caso relatado. Após a impossibilidade de concluir o diagnóstico apenas avaliando imagem da radiografia panorâmica, foi solicitado a TCFC para confirmação do diagnóstico final, facilitando também o planejamento do procedimento cirúrgico.

A literatura reporta várias opções de tratamento para fusão dentária, sendo a terapia endodôntica e a remoção cirúrgica as mais comuns. Normalmente o tratamento endodôntico é complicado em molares fundidos devido à complexidade anatômica dos canais radiculares (Ley et al., 2019). Diante disso, os fatores que levaram a escolha do tratamento realizado foram a paciente não apresentar o elemento erupcionado, e nem espaço suficiente na arcada dentária, o que impossibilita a terapia endodôntica. 
Ley et al. (2019) afirmam que quando ocorre a fusão entre um dente normal e um dente supranumerário, é necessário um espaço maior na maxila ou mandíbula.

Xinjia Cai et al. (2020) afirmam em seu relato de caso que molares fusionados geralmente são removidos cirurgicamente devido a sua anatomia e localização complicada. Park et al. (2020) relata em seu artigo, a importância da extração precoce de dentes supranumerários com formas anômalas, pois o momento da intervenção cirúrgica é crucial para o sucesso do tratamento. Diante disso, o tratamento proposto e realizado foi a exodontia, pois os elementos envolvidos apresentavam-se localizados na região vestibular do segundo molar inferior e próxima ao canal mandibular, mesmo com rizogênese incompleta. Então, caso o dente fosse mantido até sua rizogênese completa, haveria maiores chances de lesão ao NAI, e outras complicações durante a exodontia, devido sua impactação, como chance de fratura mandibular, por causa da necessidade de maior desgaste ósseo na região vestibular.

No caso clínico apresentado por Ferreira-Júnior et al. (2014), o paciente apresentou um quadro de parestesia do NAI, que perdurou por 15 dias, após a cirurgia de um terceiro molar fusionado e impactado, onde o dente precisou ser seccionado em três fragmentos para ser extraído com sucesso, o que confirma o exposto anteriormente de que a abordagem precoce nesses casos evita maiores complicações, uma vez que, a paciente do caso relatado neste trabalho não apresentou nenhuma queixa pósoperatória.

\section{Conclusão}

Após a revisão da literatura foi possível observar que o presente caso clínico é um raro exemplo de elemento supranumerário fusionado com dente permanente em região posterior da mandíbula, onde o elemento foi extraído integralmente de forma precoce. Concluímos que o acompanhamento da evolução dos terceiros molares deve ser feito desde cedo para observar seu processo de formação e diagnosticar qualquer anomalia que venha a surgir, a fim de solucionar o quanto antes para evitar maiores consequências. Ademais, ressaltamos a importância da TCFC no diagnóstico diferencial e na obtenção de informações para o planejamento cirúrgico.

O relato apresentado é considerado raro, e por isso apresenta relevância na área da cirurgia oral, ressaltando a necessidade do diagnóstico correto e abordagem precoce para evitar maiores complicações. Em virtude disso, é importante a realização de estudos futuros que abordem informações que auxiliem no correto diagnóstico, bem como na conduta de casos semelhantes.

\section{Referências}

Báez-Quintero, L. C., Percinoto, C., Cunha, R. F., Amaral, J. G., Hosida, T. Y., Favretto, C. O., ... \& Delbem, A. C. B. (2017). OCCl o35-Mesiodente impactado na linha meia da maxila e assoalho da fossa nasal: caso clínico. Archives Of Health Investigation, 6.

Cai, X., Cui, L., \& Chen, K. (2020). Impacted Right Maxillary Third Molar Fused With Supernumerary Tooth. Journal of the College of Physicians and Surgeons--Pakistan: JCPSP, 30(5), 552.

Dias, G. F., Hagedorn, H., Maffezzolli, M. D. L., Silva, F. D. F. D., \& Alves, F. B. T. (2019). Diagnóstico e tratamento de dentes supranumerários na clínica infantil-relato de caso. Revista CEFAC, 21.

Ferreira-Júnior, Osny, ÁVILA, Luciana Dorigatti de, SAMPIERI, Marcelo Bonifácio da Silva, \& DIAS-RIBEIRO, Eduardo. (2014). Terceiro molar inferior fundido com dente supranumerário: diagnóstico e plano de tratamento por meio da tomografia computadorizada de feixe cônico. RGO - Revista Gaúcha de Odontologia, 62 (4), 453-458. https://doi.org/10.1590/1981-8637201400040000161061

García Rosales, L., Rebolledo Cobos, M., Moreno Juvinao, V., Mejía Grimaldo, D., \& de La Hoz Urrutia, L. (2020). Anomalías dentales en personas con discapacidad. Revista Cubana de Estomatología, 57(3).

Laganà, G., Venza, N., Borzabadi-Farahani, A., Fabi, F., Danesi, C., \& Cozza, P. (2017). Dental anomalies: prevalence and associations between them in a large sample of non-orthodontic subjects, a cross-sectional study. BMC oral health, 17(1), 1-7.

Ley, A. M., Viana, F. L. P., Cruz, S. M. L., \& Vasconcelos, B. C. (2019). Fused tooth: clinical approach to endodontic treatment. General dentistry, 67(6), 5961 . 
Research, Society and Development, v. 10, n. 9, e52310918303, 2021

(CC BY 4.0) | ISSN 2525-3409 | DOI: http://dx.doi.org/10.33448/rsd-v10i9.18303

Magalhaes, G. P., Paz, E. C., Silva, Y. T. C. S., de Carvalho Leite, C. M., Falcão, C. A. M., \& Ferraz, M. Â. A. L. (2019). Diagnóstico de anomalias dentárias em radiografias panorâmicas. Revista Odontológica do Brasil Central, 28(87).

Mohan, S., Kankariya, H., \& Fauzdar, S. (2012). Impacted inverted teeth with their possible treatment protocols. Journal of maxillofacial and oral surgery, $11(4), 455-457$

Moreira, K. M. S., Rezende, C. P., Silva, D. V., Imparato, J. C. P., Navarro, R. S., \& Reis, J. B. (2016). Reabilitação do incisivo central decíduo fusionado ao supranumerário: relato de caso clínico. Revista da Associacao Paulista de Cirurgioes Dentistas, 70(3), 302-305.

Neville, B. (2016). Patologia oral e maxilofacial. Elsevier Brasil.

Park, S. Y., Jang, H. J., Hwang, D. S., Kim, Y. D., Shin, S. H., Kim, U. K., \& Lee, J. Y. (2020). Complications associated with specific characteristics of supernumerary teeth. Oral surgery, oral medicine, oral pathology and oral radiology, 130(2), 150-155.

Pereira, A. S., Shitsuka, D. M., Parreira, F. J., \& Shitsuka, R. (2018). Metodologia da pesquisa científica.

Saberi, E. A., \& Ebrahimipour, S. (2016). Evaluation of developmental dental anomalies in digital panoramic radiographs in Southeast Iranian Population. Journal of International Society of Preventive \& Community Dentistry, 6(4), 291.

Shah, U. D., Patel, H., Patel, N., Ranadheer, E., \& Shoba, F. (2015). Interception in pursuit of exquisite aesthetics-a case series. Intern Journ. Of Adv. Research, $3(7), 590-8$

Silva, L. P., Salzedas, L. M. P., \& Ikuta, C. R. S. (2018). Fusão entre o dente 12 e um supranumerário: um desafio diagnóstico. Archives Of Health Investigation, 7.

Smail-Faugeron, V., Terradot, J., Bolla, M. M., \& Courson, F. (2016). Management of non-syndromic double tooth affecting permanent maxillary central incisors: a systematic review. Case Reports, 2016, bcr2016215482.

Stringhini Junior, E., Stang, B., \& Oliveira, L. B. (2015). Dentes supranumerários impactados: relato de caso clínico. Revista da Associacao Paulista de Cirurgioes Dentistas, 69(1), 89-94.

Temilola, D. O., Folayan, M. O., Fatusi, O., Chukwumah, N. M., Onyejaka, N., Oziegbe, E., ... \& Agbaje, H. (2014). The prevalence, pattern and clinical presentation of developmental dental hard-tissue anomalies in children with primary and mix dentition from Ile-Ife, Nigeria. BMC oral health, 14(1), 1-8.

Vinothkumar, T. S., Kandaswamy, D., Arathi, G., Ramkumar, S., \& Felsypremila, G. (2017). Endodontic Management of Dilacerated Maxillary Central Incisor fused to a Supernumerary Tooth using Cone Beam Computed Tomography: An Unusual Clinical Presentation. The journal of contemporary dental practice, $18(6), 522-526$ 